\title{
Sternal Involvement in Anterior Mediastinal Seminoma - A Rare Presentation
}

Rajasbala Pradeep Dhande ${ }^{1}$, Shirish V. Vaidya ${ }^{2}$, Megha Manoj ${ }^{3}$, Rohan Kumar Singh ${ }^{4}$, Harshith Gowda K.B. ${ }^{5}$

1,2,3,4,5 Department of Radiodiagnosis, Jawaharlal Nehru Medical College, Datta Meghe

Institute of Medical Sciences (DMIMS), Sawangi (Meghe), Wardha, Maharashtra, India.

\section{INTRODUCTION}

Primary malignant extra gonadal germ cell tumours (GCT) of mediastinum are very rare tumours, comprising $1-4 \%$ of all mediastinal tumours. ${ }^{1}$ Even though they are rare tumours of mediastinum, they should always be considered in the differential diagnosis of mediastinal tumours in young adults. Primary malignant GCT can be divided into 2 groups: Seminomatous and non-seminomatous tumours. Mature teratomas (non-seminomatous) are the most common primary malignant germ cell tumours of mediastinum. ${ }^{2}$ Non-seminomatous tumours tend to be more aggressive than seminomatous tumours present as heterogeneous masses with areas of necrosis and show local invasion. Seminomas usually present as lobulated homogeneous masses and rarely show signs of local invasion. These tumours usually present with asymptomatic lesions or with symptoms due to compression of adjacent structures and usually have a better prognosis than non-seminomatous tumours. Here, we present a case of primary malignant extra gonadal seminoma with sternal involvement.

\section{PRESENTATION OF CASE}

A 42-year-old man presented with a chief complaint of a swelling in the anterior chest wall which was increasing gradually over a span of 2 years. There was no significant past medical or surgical history. On local examination, a large firm swelling of size of $10 \times 10 \mathrm{~cm}$ was found in the sternal region.

Systemic examination was unremarkable. Patient was admitted in the Department of Surgery for further evaluation of the same. As a preliminary investigation, a chest radiograph was taken, which showed a well-defined opacity in the mediastinal region (Figure 1).
Corresponding Author: Dr. Rohan Kumar Singh, Junior resident, Department of Radiodiagnosis, Jawaharlal Nehru Medical College, Datta Meghe Institute of Medical Sciences (DMIMS), Sawangi (Meghe), Wardha, Maharashtra, India.

E-mail:

rohankumarsingh2222@gmail.com

DOI: $10.14260 /$ jemds $/ 2021 / 371$

How to Cite This Article:

Dhande RP, Vaidya SV, Manoj M, et al. Sternal involvement in anterior mediastinal seminoma - a rare presentation. J Evolution Med Dent Sci 2021;10(23):1799-1802, DOI: 10.14260/jemds/2021/371

Submission 08-02-2021, Peer Review 13-04-2021, Acceptance 21-04-2021, Published 07-06-2021.

Copyright (C) 2021 Rajasbala Pradeep Dhande et al. This is an open access article distributed under Creative Commons Attribution License [Attribution 4.0 International (CC BY 4.0)] 


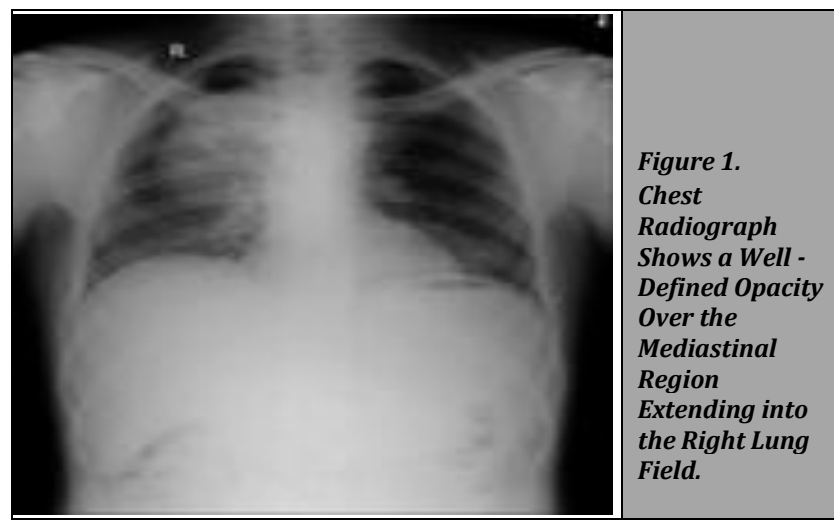

Later the patient was advised FNAC of the lesion for further evaluation which revealed a serous cystic lesion with chronic inflammation. Following this the patient underwent surgical excision of the lesion and the sample was sent for histopathological analysis. On histopathological evaluation, the lesion was found to be Seminoma (Extra gonadal germ cell tumour). In view of the histopathology report, a CECT thorax was advised by the tumour board which revealed a large illdefined heterogeneously enhancing anterior mediastinal mass with calcific foci (Figure 2). No other primary malignancy was identified on further evaluation.

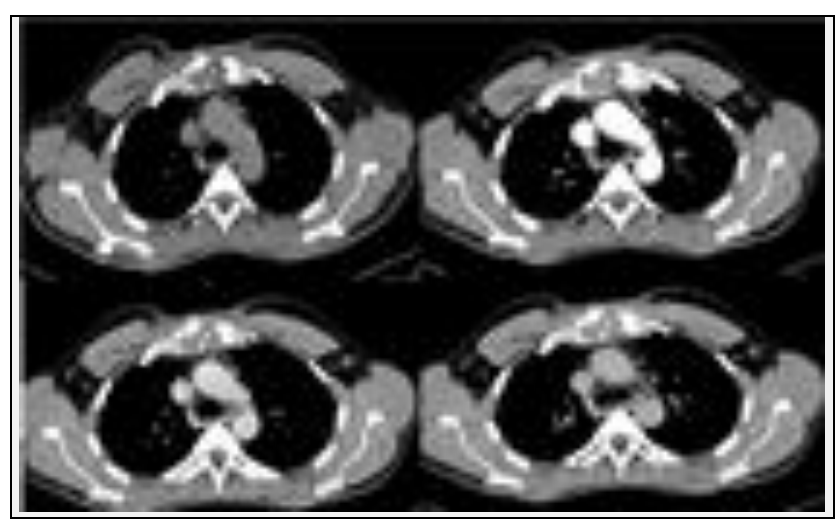

Figure 2. Axial NCCT and CECT Images of Upper Thorax Show an III Defined, Heterogeneously Enhancing Mass with Calcific Foci in the Prevascular Region. The Mass is Seen Causing Erosive Destruction of the Sternum

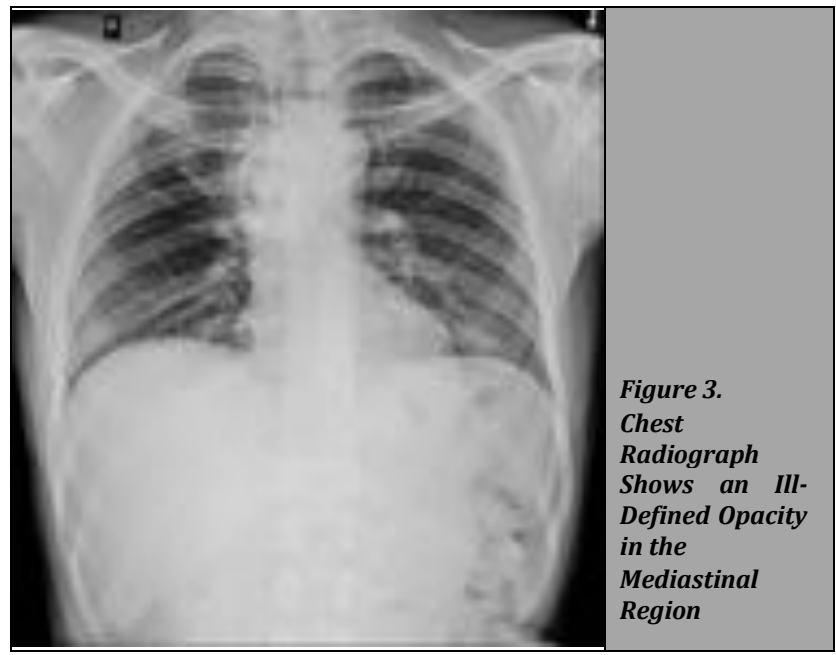

For further management, the case was discussed in the Tumour board and the patient was advised 4 cycles of EP regimen and 6 months follow up. 2 weeks after the procedure, patient was started on Inj. cisplatin $125 \mathrm{mg}$ and Inj. etoposide $249 \mathrm{mg}$, every 21 days for 4 months. No radiotherapy was given as the patient was not willing for the same. Patient underwent 3 cycles of chemotherapy and did not come for a 6 month follow up, but presented with the same complaint after 1 year.

Chest radiograph taken after 1 year showed an ill-defined opacity in the mediastinal region (Figure 3). A CECT thorax was done to assess if any recurrence of the lesion was present and determine the extent of the lesion. CECT thorax revealed, a well-defined lobulated mass in the pre-vascular region causing erosive destruction of the sternum and extension into the subcutaneous plane (Figure 4).

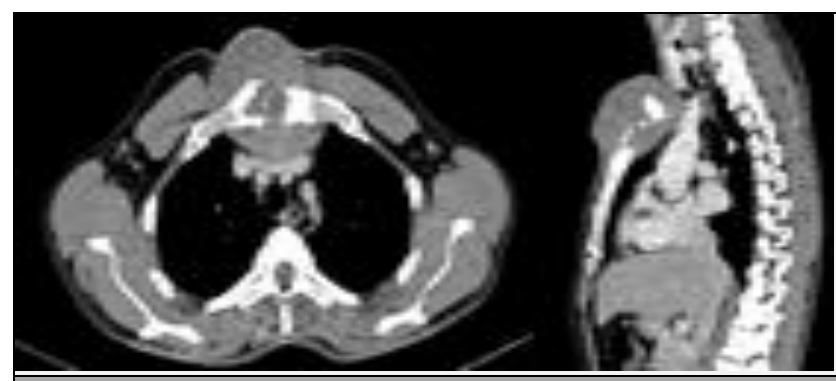

Figure 4. Axial and Sagittal CECT Images Show a Lobulated Mass in the Anterior Mediastinum Causing Destructive Erosion of the Sternum and Extension into the Subcutaneous Plane. The Mass Can be Seen Abutting the Right Brachiocephalic Vein with No Obvious Erosion

The patient was advised 3 cycles of chemotherapy with VbIP regimen. He was given the $1^{\text {st }}$ dose of Inj. cisplatin, Inj. ifosfamide and Inj. etoposide and discharged. The patient did not come for further treatment.

\section{DISCUSSION}

Anterior mediastinal tumours include a wide variety of primary and secondary lesions, ranging from thymoma to thoracic aorta aneurysms. The anterior mediastinum is bounded anteriorly by sternum and, posteriorly by ascending aorta, brachiocephalic vessels, and pericardium. Superiorly, it extends till the thoracic inlet and inferiorly till the diaphragm. Its contents include thymus, lymph nodes, fat and may also contain retrosternal thyroid. Anterior mediastinal masses usually arise from these contents. Common mediastinal masses include thymomas, thyroid masses, lymphomas, thoracic aortic aneurysms and germ cell tumours. Secondary tumours are more commonly seen than primary tumours and include metastasis from lung, oesophagus, and testis. ${ }^{3}$

Mediastinal tumours do not have age predilection and can occur at any age. Most of the mediastinal masses are clinically silent and are found incidentally. But they can also present with symptoms like cough or difficulty in breathing, hoarseness of voice or chest pain dysphagia and fever. They can also present with symptoms of SVC compression. Rarely, they can present as an external mass. Davis et al. found that malignant tumours were more symptomatic that benign lesions. ${ }^{4}$ An increased incidence of mediastinal germ cell tumours has been noted in patients with Klinefelter's syndrome. 5 
Initial evaluation of mediastinal tumours is done by performing a chest radiograph in anteroposterior and lateral views. On a chest radiograph, anterior mediastinal masses give a positive hilum overlay sign and silhouettes cardiac margin. A contrast enhanced CT or MRI is then performed to characterise the lesion and also to evaluate the extent of the lesion. Additional PET scans can also be done to monitor patient's response to treatment. Raised serum alpha fetoprotein, beta human chorionic gonadotropin (HCG) and lactate dehydrogenase (LDH) may be raised in germ cell tumours.

Extra gonadal germ cell tumours (GCT) are a rare group of tumours, accounting for only about $1-5 \%$ of germ cell tumours. ${ }^{6}$ They arise from primitive germ cells that have failed to migrate during embryonic period. They can be seen anywhere along the midline starting from pineal gland to presacral region. Mediastinum is the most common extra gonadal site for these tumours, comprising about $50-70 \%$ of extra gonadal GCTs. ${ }^{7}$ They are typically seen in men in their $3^{\text {rd }}$ to $4^{\text {th }}$ decade and are most commonly seen in the anterior mediastinum, but they can also involve the middle mediastinum. Incidence of benign GCTs is same in both males and females, although malignant GCTs are more common in males. Primary GCT of anterior mediastinum is very rare. Primary malignancy of testicles and retroperitoneal metastases have to be ruled out to diagnose primary extra gonadal germ cell tumours. Primary testicular tumour rarely metastasizes to mediastinum in the absence of retroperitoneal tumour. ${ }^{8}$ Liu et al. found that seminomas comprised of $33 \%$ of all primary malignant mediastinal GCT, followed by yolk sac tumours and mixed tumours. ${ }^{9}$ Other GCTs include teratoma, embryonal cell carcinoma, yolk sac tumour and choriocarcinoma.

Mediastinal seminomas are less commonly seen than nonseminomatous germ cell tumours. They are slow growing tumours that usually present as large well-defined lobulated homogeneous mass, showing mild enhancement on CECT. Haemorrhage, cysts and calcifications are seen rarely. It rarely causes local invasion and usually metastasize to lymph node and causes a mass effect on Superior vena cava. It typically extends posterior or laterally to either side. On MRI, it appears isointense to hypointense on $\mathrm{T} 1$ and $\mathrm{T} 2$ weighted images and shows homogeneous enhancement on post contrast study. It usually causes mass effect on the adjacent structures rather than invade them. Metastasis has been reported in about $40 \%$ cases with poor prognosis in extra-pulmonary metastases. ${ }^{10}$ Metastases most commonly occur to the lungs and intrathoracic lymph nodes followed by the skeletal system. Few seminomas have shown metastases to liver, spleen, adrenal glands, and brain.

Primary malignancy involving the sternum is uncommon. Metastases and secondary extension of mediastinal masses or thyroid is more common than primary neoplasms. Neoplastic involvement of sternum is a bad prognostic marker which can significantly alter the choice of treatment. CT is the modality of choice to evaluate these lesions due to its high contrast and spatial resolution. ${ }^{11}$ To our knowledge, there has been no reported case of a primary anterior mediastinal seminoma causing erosion of sternum.

Histologically seminomatous tumour consists of medium sized round to oval cells with eosinophilic cytoplasm and round to oval nuclei.12 Mediastinal germ cell tumours have a poor prognosis compared to their testicular counterparts.
Seminomatous tumours respond relatively better to cisplatin based chemotherapy and radiotherapy than nonseminomatous germ cell tumours. ${ }^{13}$ A multimodality approach involving radiological imaging, surgery, pathological correlation, and oncology is required for appropriate management of these patients. Surgery followed by chemotherapy and radiotherapy is the ideal approach in management of malignant seminomas. Patients have to be kept on regular follow up to assess for recurrence.

Radiological differential diagnoses in our case include Chondrosarcoma and Osteosarcoma of sternum. Chondrosarcoma is the most common primary bone tumour which affects the sternum. A large soft tissue component and rapid enlargement is seen in these lesions. Osteosarcoma of sternum is a very rare tumour of sternum that can present with similar imaging features. It can be primary or secondary tumours. They are seen in slightly older patients and have a bad prognosis.

\section{CONCLUSIONS}

Malignant primary seminomatous tumour of mediastinum is a rare entity. We would recommend the radiologists to consider these lesions in their imaging evaluation, especially in young adults. Radiological imaging and serum markers together will help us immensely to clench the diagnosis of germ cell tumours accurately. Both can also be used to assess a patient's response to treatment and a possible recurrence, which is of utmost importance in managing these patients.

Financial or other competing interests: None.

Disclosure forms provided by the authors are available with the full text of this article at jemds.com.

\section{REFERENCES}

[1] Gu L, Zhang L, Hou N, et al. Clinical and radiographic characterization of primary seminomas and nonseminomatous germ cell tumors. Niger J Clin Pract 2019;22(3):342-9.

[2] Strollo DC, De-Christenson MLR. Primary mediastinal malignant germ cell neoplasms: imaging features. Chest Surg Clin N Am 2002;12(4):645-58.

[3] Govindan R, Devita VT. Devita, Hellman and Rosenberg's cancer: principles \& practice of oncology review. Lippincott Williams \& Wilkins 2009.

[4] Davis RD Jr, Oldham HN Jr, Sabiston DC Jr. Primary cysts and neoplasms of the mediastinum: recent changes in clinical presentation, methods of diagnosis, management and results. Ann Thorac Surg 1987;44(3):229-37.

[5] Völkl TM, Langer T, Aigner T, et al. Klinefelter syndrome and mediastinal germ cell tumors. Am J Med Genet A 2006;140(5):471-81.

[6] Shinagare AB, Jagannathan JP, Ramaiya NH, et al. Adult extra gonadal germ cell tumors. AJR Am J Roentgenol 2010;195(4):W274-80.

[7] Gao Y, Jiang J, Liu Q. Extragonadal malignant germ cell tumors: a clinicopathological and immunohistochemical 
analysis of 48 cases at a single Chinese institution. Int J Clin Exp Pathol 2015;8(5):5650-7.

[8] Levitt RG, Husband JE, Glazer HS. CT of primary germ-cell tumors of the mediastinum. AJR Am J Roentgenol 1984;142(1):73-8.

[9] Liu Y, Wang Z, Peng ZM, et al. Management of the primary malignant mediastinal germ cell tumors: experience with 54 patients. Diagn Pathol 2014;9(1):1-6.

[10] Travis WD, Brambilla E, Burke AP, et al. Introduction to the 2015 World Health Organization classification of tumors of the lung, pleura, thymus and heart. J Thorac Oncol 2015;10(9):1243-60.

[11] Singh A, Chandrashekhara SH, Triveni GS, et al. Imaging in sternal tumours: a pictorial review. Pol J Radiol 2017;82:448-56.

[12] Kalhor N, Moran CA. Primary germ cell tumors of the mediastinum: a review. Mediastinum 2018;2:4.

[13] Zhou ZT, Wang JW, Yang L, et al. Primary germ cell tumor in the mediastinum-report of 47 cases. Zhonghua Zhong Liu Za Zhi 2006;28(11):863-6. 